\title{
Asteroseismology of HADS stars: V974 Oph, a radial pulsator flavoured by nonradial components $\star$
}

\author{
E. Poretti ${ }^{\star \star}$ \\ INAF-Osservatorio Astronomico di Brera, Via Bianchi 46, 23807 Merate, Italy \\ Received 5 May 2003/ Accepted 7 July 2003

\begin{abstract}
The analysis of a dense time-series on V974 Oph disclosed the rich pulsational content (at least five independent terms) of this high-amplitude ( $0.60 \mathrm{mag}$ in $B$-light) $\delta$ Sct star. A mode with a frequency very close to the main one (probably the fundamental radial mode) has been detected: such a doublet is not a common feature in stars of the same class. Also another term can be considered a radial one, but the high ratio (0.786) raises some problems that can be solved only by admitting very low metallicity. It is quite evident that some undetectable terms are again hidden in the noise, as the least-squares fit leaves a rms residual much higher than the observational noise. All that considered, nonradial modes seem to play a key rôle in the light variability of V974 Oph. Revealing an unsuspected asteroseismic interest, V974 Oph provides a link between low- and high-amplitude $\delta$ Sct stars.
\end{abstract}

Key words. methods: data analysis - stars: oscillations - techniques: photometric - stars: variables: $\delta$ Sct stars: individual: V974 Oph

\section{Introduction}

In one of the first reviews on $\delta$ Sct and related stars, Breger (1979) proposed to drop the distinction between high- and small-amplitude pulsators located on the lower part of the instability strip. This suggestion was applied by the GCVS, which simplified the old nomenclature (RRs, Dwarf Cepheids, AI Vel, $\delta$ Sct, ...) only maintaining the subdivision between variables belonging to the Pop. I ( $\delta$ Sct stars, DSCT) and to the Pop. II (SX Phe variables, SX PHE). This simplification was widely accepted, as at that time the amplitude was not considered a physical discriminant between $\delta$ Sct stars: the light curves of BN Cnc and AD CMi shown by Breger (1979, his Fig. 1) are very similar in shape, but very different in amplitude ( 0.014 and $0.294 \mathrm{mag}$, respectively), suggesting a different energy in the same physical process.

The very strong observational effort made in the $80 \mathrm{~s}$ and $90 \mathrm{~s}$ demonstrated how small amplitude DSCT stars show a large variety of nonradial modes and how their light variability is complex (multiperiodicity, close doublets of frequencies, amplitude variations, ...). They seem to be very different from high-amplitude DSCT stars, which are classical radial pulsators, most monoperiodic. Therefore, the subclass of the High Amplitude Delta Scuti (HADS) stars has been introduced; their light curves have an asymmetrical shape which can be quantified by Fourier decomposition. A review of the HADS

\footnotetext{
* Based on observations collected at Europan Southern Observatory, La Silla, Chile

$\star \star$ e-mail: poretti@merate.mi.astro.it
}

properties is given by McNamara (2000): it seems that HADS are concentrated in the central part of the instability strip, in a well-defined region. At first glance, the rough subdivision between radial, mostly monoperiodic (double-mode in some cases, but always radial modes) HADS stars and mostly nonradial, multiperiodic low-amplitude stars seems valid. The acronym LADS has sometimes been used to identify the latter variables, which are also considered as the more promising asteroseismic targets. However, further investigations and observational studies have modified this idyllic picture. Walraven et al. (1992) found that AI Vel is a multiperiodic variable, probably in the fundamental and first three overtone radial modes; they also suggest that a nonradial mode can be present in the light curve of this HADS star. Later, Garrido \& Rodríguez (1996) analyzed some time-series of HADS stars, finding that in the cases of SX Phe and DY Peg other radial and nonradial modes could be excited. More recently, Arentoft et al. (2001) found evidence both of amplitude variation and of excitation of nonradial modes in the light curve of V1162 Ori; the same result has been obtained by Zhou (2002) on AN Lyn. Multimode SX Phe variables have been found among the blue stragglers of NGC 3201 (Mazur et al. 2003).

Therefore, nowadays the phenomenology of HADS stars appears more similar to that of small-amplitude $\delta$ Sct stars. The only remarkable difference is that the nonradial modes in HADS stars have a much smaller amplitude (order of magnitudes) than the radial modes and that period and amplitude variations can be considered as small perturbations of a mode always clearly visible in the light curve. In this paper we 


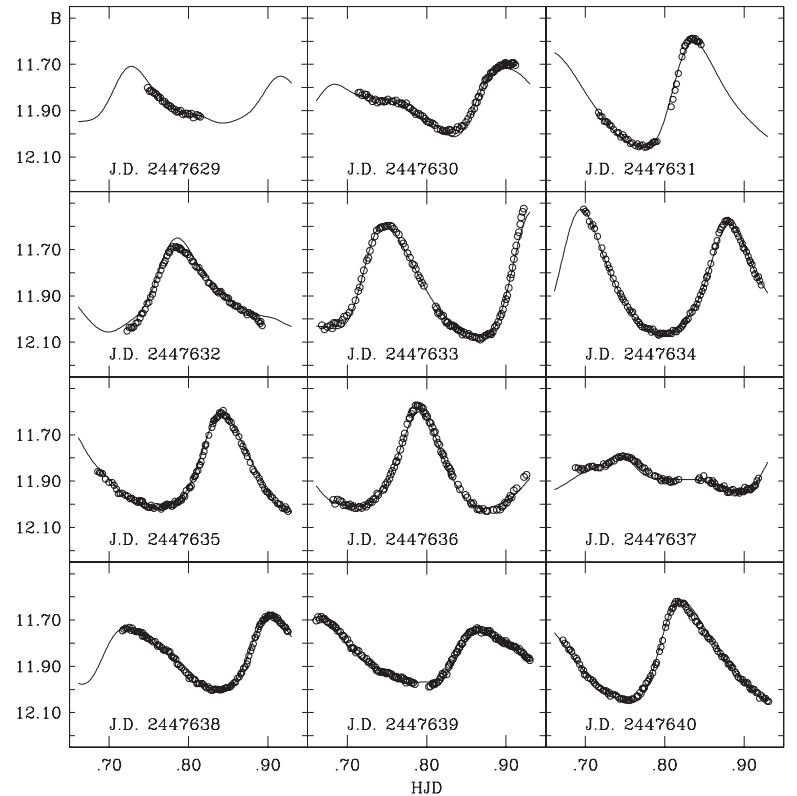

Fig. 1. Light curves of V974 Oph in standard $B$ magnitudes. Solid line represents the 12-terms fit.

discuss the case of V974 Oph, which seems to make even closer to each other the phenomenology of HADS and LADS stars.

\section{Observations}

V974 Oph was monitored on 13 consecutive nights from April 11 to 23, 1989 as a target observed at the end of nights devoted to HD 101158 (Poretti 1991). Measurements were performed at the ESO 50-cm telescope (La Silla, Chile - now decommissioned) by using the EMI 9789QB photomultiplier. HD 159822 has been used as comparison star and the transformation into the standard system (by measurements of four stars in the E7 region) supplied $V=10.08 \pm 0.01, B=10.23 \pm 0.01$. We obtained 1150 points on V974 Oph in $B$-light; the 528 measurements of the check star HD 160108 yielded a standard deviation of 0.0041 mag in $B$-light.

The observational history of V974 Oph is summarized by Poretti \& Antonello (1998). The possibility that V974 Oph is similar to V1719 Cyg and V798 Cyg is ruled out when looking at the light curves obtained in this observing run (Fig. 1).

\section{Frequency analysis}

The least-squares power spectrum method (Vaniček 1971) allowed us to detect one by one the constituents of the light curve. After each detection, we refined the frequency values by applying the MTRAP code (Carpino et al. 1987) and they are introduced as known constituents (KCs) in the new search. Such a procedure is particularly suitable for a multimode, highamplitude pulsation as it keeps the relationships between the detected terms (i.e., $2 f_{1}, f_{1}+f_{2}, \ldots$ ) locked and it doesn't use the data prewhitening, as amplitudes and phases of the KCs are recalculated for each trial frequency.

The analysis of the time-series evidenced the large contribution of the $f_{1}$ term, i.e., $5.2336 \mathrm{~d}^{-1}$ (Fig. 2, top row, left

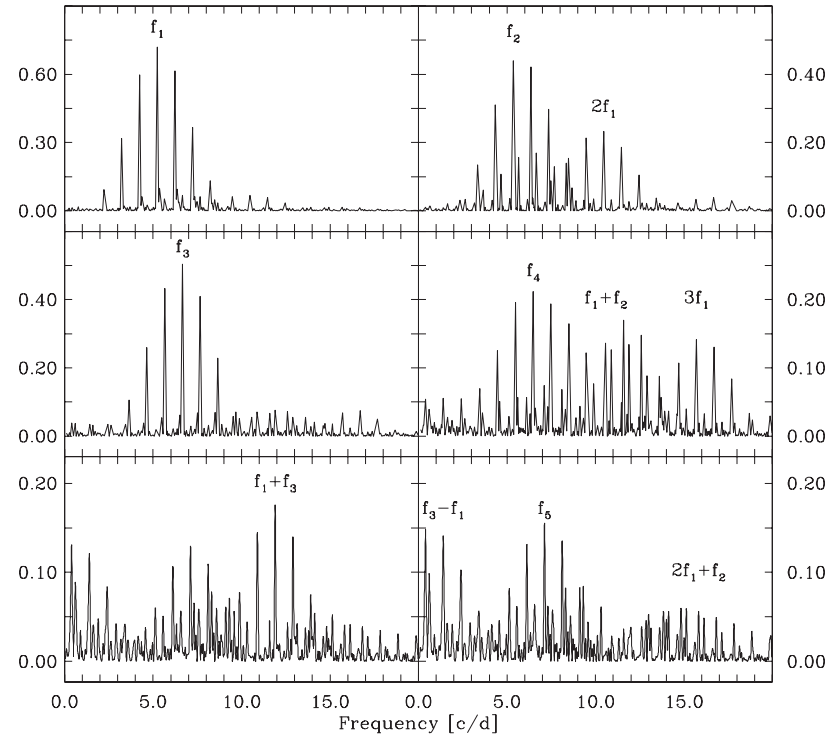

Fig. 2. Power spectra of the V974 Oph data.

panel). This periodicity is characterized by a strong asymmetric light curve and the harmonics $2 f_{1}$ (top row, right panel) and $3 f_{1}$ (middle row, right panel) are detected going further in the analysis. However, the second contribution in amplitude comes from the $f_{2}=5.3498 \mathrm{~d}^{-1}$ term (top row, right panel), i.e., a periodicity located close to $f_{1}$, just at the resolution limit of our dataset. It should be noted that the alias at $6.3498 \mathrm{~d}^{-1}$ is only slightly lower. Since its amplitude is very large (0.07 mag), we can verify if the preference for the $5.3498 \mathrm{~d}^{-1}$ term with respect to the $6.3498 \mathrm{~d}^{-1}$ one is justified: indeed, when introducing the latter value as k.c., we recover a peak at the former, confirming that our first choice is the right one. However, there is another problem: being located at the resolution limit, we cannot be sure that $5.3498 \mathrm{~d}^{-1}$ is the exact value as its value can be modified by the interaction with $f_{1}$.

The other peaks clearly detectable in the power spectra are $f_{3}=6.6599 \mathrm{~d}^{-1}$ and $f_{4}=6.4762 \mathrm{~d}^{-1}$ (middle row, left and right panels). Both these peaks show a symmetric alias structure in the spectra: also for them, including an alias as k.c. yields a residual signal at the central value, detectable later in the frequency analysis.

In the fourth power spectrum we can also detect the structure related to the $11.58 \mathrm{~d}^{-1}$ term; this term is the alias at $+1 \mathrm{~d}^{-1}$ of $f_{1}+f_{2}$ and its predominance over the expected value is not significant. Indeed, two steps beyond, the $2 f_{1}+f_{2}$ term is detected in the sixth power spectrum (bottom row, right panel).

The fifth power spectrum (bottom row, left panel) provides a good confirmation of the previous detections, as the highest peak is at $f_{1}+f_{3}=11.8935 \mathrm{~d}^{-1}$ and the $f_{3}-f_{1}=$ $1.4236 \mathrm{~d}^{-1}$ term is also visible, i.e., we detected both the coupling terms between $f_{1}$ and $f_{3}$. The last term we can find in the time-series is $f_{5}=7.1206 \mathrm{~d}^{-1}$, i.e., the highest peak in the sixth spectrum.

Looking at Fig. 1 we can expect that a conspicuous set of frequencies is necessary to fit the light curves in a satisfactory way. We can propose five independent frequencies $\left(f_{1}=\right.$ $5.2336 \mathrm{~d}^{-1}, f_{2}=5.3498 \mathrm{~d}^{-1}, f_{3}=6.6599 \mathrm{~d}^{-1}, f_{4}=6.4762 \mathrm{~d}^{-1}$, 
Table 1. Parameters of the least-squares fit of the measurements on V974 Oph. $T_{0}=$ HJD 2447628.000 .

\begin{tabular}{lcccc}
\hline \hline Term & $\begin{array}{r}\text { Freq. } \\
{\left[\mathrm{d}^{-1}\right]}\end{array}$ & $\begin{array}{r}\text { Ampl. } \\
{[\mathrm{mag}]}\end{array}$ & $\begin{array}{c}\text { Phase } \\
{[\mathrm{rad}]}\end{array}$ & $f_{1} / f_{N}$ \\
\hline$f_{1}$ & 5.2336 & 0.1587 & 2.55 & 1.000 \\
$2 f_{1}$ & & 0.0448 & 2.55 & \\
$3 f_{1}$ & & 0.0151 & 2.75 & \\
$4 f_{1}$ & & 0.0049 & 3.02 & \\
$f_{2}$ & 5.3498 & 0.0696 & 1.27 & 4.41 \\
$f_{1}+f_{2}$ & & 0.0149 & 4.36 & \\
$2 f_{1}+f_{2}$ & & 0.0062 & 4.82 & \\
$f_{3}$ & 6.6599 & 0.0427 & 5.96 & 0.786 \\
$f_{1}+f_{3}$ & & 0.0119 & 6.02 & \\
$f_{3}-f_{1}$ & & 0.0079 & 4.53 & \\
$f_{4}$ & 6.4762 & 0.0171 & 4.13 & 0.807 \\
$f_{5}$ & 7.1206 & 0.0104 & 0.61 & 0.734 \\
\hline \multicolumn{3}{l}{ Mean $B$ magnitude } & $11.871 \pm 0.001$ & \\
Residual rms & \multicolumn{4}{c}{$0.0141 \mathrm{mag}$} \\
Measurements & \multicolumn{3}{c}{} \\
\hline \multicolumn{4}{l}{}
\end{tabular}

$\left.f_{5}=7.1206 \mathrm{~d}^{-1}\right), 3$ harmonics of $f_{1}$ and some coupling terms between $f_{1}, f_{2}$ and $f_{3}$. Table 1 summarizes the least-squares solution (shown also in Fig. 1) of the $B$ magnitudes: a cosine series has been used. Formal errors have been calculated following Montgomery \& O'Donoghue (1999): they are around $4 \times 10^{-4}$ mag on the amplitudes, $1-3 \times 10^{-3} \mathrm{~d}^{-1}$ on the frequencies of smaller amplitude, $10^{-4} \mathrm{~d}^{-1}$ on the frequencies of larger amplitude, $10^{-2}$ rad on the phases.

However, the least-squares solution is far from being considered as complete. The residual $\mathrm{rms}$ is $0.015 \mathrm{mag}$, a value 3.6 higher than the observational error. However, the residual rms doesn't decrease rapidly by adding new terms: indeed, we get a rms residual of $0.0103 \mathrm{mag}$ by introducing $18 \mathrm{KCs}, 0.0086 \mathrm{mag}$ by 24 k.c.'s, 0.0074 mag by $30 \mathrm{KCs}$. A huge number of terms is necessary to reduce the rms residual to the noise level, i.e., the 0.0041 mag obtained from the measurements of the check star.

The power spectrum obtained considering the 12 terms of Table 1 as KCs is shown in Fig. 3. Residual signal is present in different parts $\left(f<2 \mathrm{~d}^{-1}, f \approx 9 \mathrm{~d}^{-1}, f \approx 13 \mathrm{~d}^{-1}\right)$, but no peak reaches the acceptance levels $(S / N=4.0$ for independent terms, $S / N=3.5$ for harmonics or combination terms; Kuschnig et al. 1997). As regards the coupling term $f_{2}-f_{1}=0.1162 \mathrm{~d}^{-1}$ we note that it is close to the resolution attainable with our time baseline and therefore its non-detection is not surprising.

Figure 4 shows the residuals between the measurements and the fitting curve in few representative cases. Most of the residual scatter mimics high frequency variations, but the signal seems confined in the low-frequency region (see the insert in Fig. 3). As a matter of fact, the power spectrum does not show any peak at all for $f>25 \mathrm{~d}^{-1}$. A possible explanation is the presence of other terms, not resolved from the detected ones. In such a case, the least-squares solution can be locally unsatisfactory.

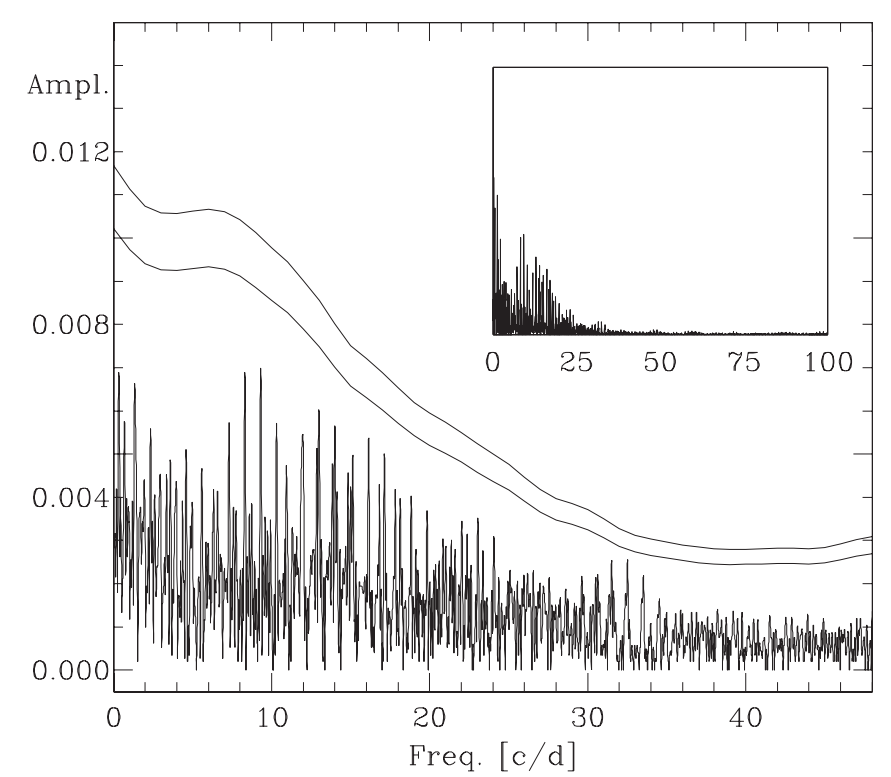

Fig. 3. Residual amplitude spectrum after having considered 12 known constituents. Amplitudes are in mag. The upper line denotes $S / N=$ 4.0, the lower line denotes $S / N=3.5$. The insert displays the power spectrum extended as far as $100 \mathrm{~d}^{-1}$.

The residuals of JD 2447632 are systematically shifted $(-0.03 \mathrm{mag})$ respect with the zero level. This fact can suggest an instrumental effect, but the measurements of the check star don't show any relevant peculiarity. We also tried to fit the data by introducing individual night corrections, but these arbitrary shifts didn't contribute to decrease rapidly the residuals.

\section{Discussion}

The only tool we have to investigate the pulsational content of V974 Oph is the ratio between the different frequencies. Recent observational studies allow us to extend the same analysis to other HADS stars. Figure 5 shows the frequency content of V974 Oph compared to AI Vel (Walraven et al. 1992), V1162 Ori (Arentoft et al. 2001), V567 Oph (Kiss et al. 2002), AN Lyn (Zhou 2002), SX Phe and DY Peg (Garrido \& Rodríguez 1996), and MACHO stars (Alcock et al. 2000a). As regards these latter stars, we considered only confirmed multimode pulsators (i.e., stars listed in Table 4A in Alcock et al. 2000a, but not those in Table 4B). In the figure we plot the observed $f_{F} / f_{i}$ ratio, assuming that the longest period is the fundamental radial mode; the only exceptions are SX Phe and MACHO 109.20634.24, where the longest period is a small amplitude one, assumed to be a nonradial mode. We also note that Garrido \& Rodríguez (1996) are rather cautious on the reality of this period in the SX Phe data. The canonical double-mode radial pulsators have not been considered in Fig. 5: they provide the well-known 0.77 ratio between the fundamental $(F)$ and the first overtone (1O) radial mode, shown as a vertical line. On the other hand, double-mode pulsators with $\mathrm{F} / 1 \mathrm{O}$ ratios slightly different from 0.77 have been reported to show how these discrepancies are currently observed (see Sect. 4.2). The line corresponding to the $\mathrm{F} / 2 \mathrm{O}$ ratio (i.e., $0.620 ; 2 \mathrm{O}$, second overtone radial mode) is also traced in Fig. 5. We have to note that the presence of 


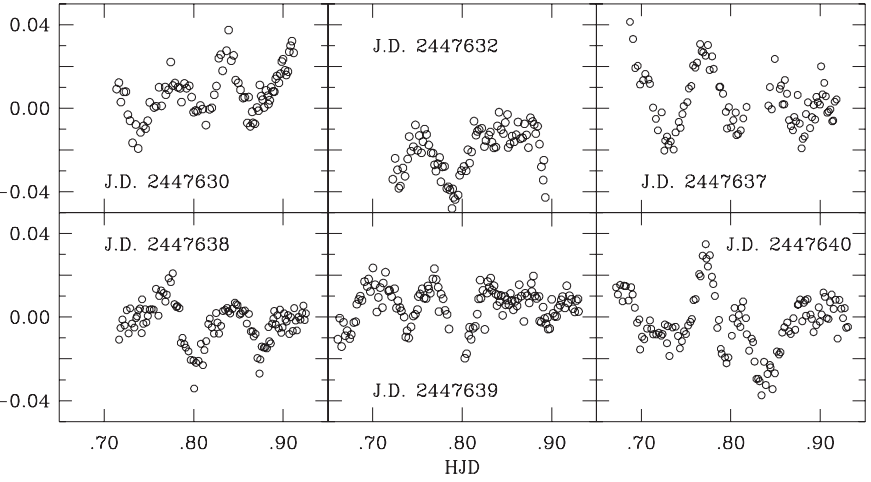

Fig. 4. Residual after subtracting the 12-term solution from the original measurements.

small amplitude, nonradial modes in the light curves of some stars has been established on the basis of very different observational conditions (time sampling, data homogeneity) and therefore they need a more stringent observational confirmation.

The full amplitude of the V974 Oph light curve is $0.60 \mathrm{mag}$ in $B$-light, which scales down to $0.43 \mathrm{mag}$ in $V$ light. Among the stars considered here, only AI Vel and MACHO 121.22427.551 (a canonical $\mathrm{F} / 1 \mathrm{O}$ double-mode pulsator) have a higher amplitude (0.67 and 0.60, respectively); SX Phe and V567 Oph have similar amplitudes, while the other stars are around $0.20 \mathrm{mag}$. It should be noted that V974 Oph has the longest period, SX Phe the shortest $(0.055 \mathrm{~d})$. Therefore, the sample is quite heterogeneous, also considering that SX Phe is for sure a Pop. II star, while others are more probably Pop. I stars.

\subsection{The $f_{1}-f_{2}$ doublet}

Close doublets of frequencies were recently found in different classes of high-amplitude pulsators. They are common in RR Lyr stars, both fundamental (RRab, Moskalik \& Poretti 2003) and first-overtone radial pulsators (RRc stars, Alcock et al. 2000b). There are also five well-defined examples (V1, V3, V4, V6 and V10) of close doublets among the eleven SX Phe stars discovered in NGC 3201 (Mazur et al. 2003): the distribution of the frequency ratios is well sharpened around 0.980 , with the only exception of the unclear case of V3.

In the case of V974 Oph we observe $f_{1} / f_{2}=0.976$, even if the $f_{2}$ term can be affected by the limited frequency resolution (see Sect. 3). A similar doublet (0.982) has been found in the HADS star V1162 Ori (Fig. 5). The frequency resolution of MACHO data appears very suitable to detect close frequencies, but only MACHO 109.20634.24 shows a doublet composed of the period of the F-mode and one slightly longer. Close pair of frequencies are quite common among low-amplitude $\delta$ Sct stars (Breger \& Bischof 2002): however, in these stars it is not possible to assess the radial nature of one of the modes, owing to the large number of detected modes. No close doublet has been detected in the light curve of AI Vel. The most plausible explanation for this is the excitation of a nonradial mode by resonance with the main period of oscillation.

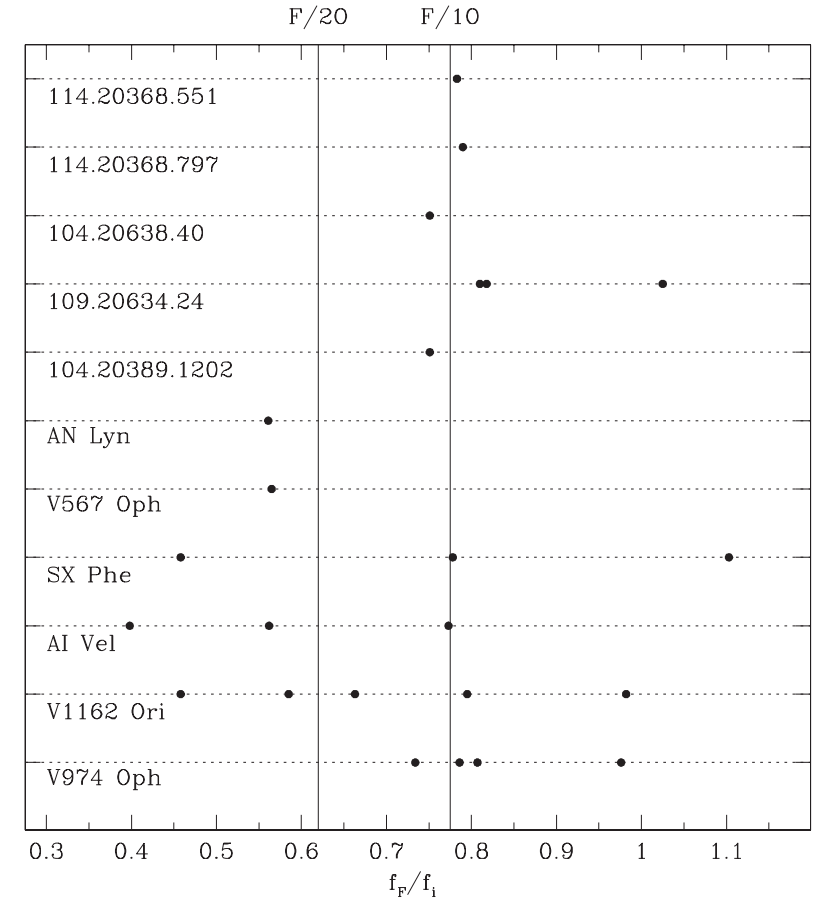

Fig. 5. Observed (filled dots) frequency ratios among HADS stars. Canonical F/1O pulsators are not shown. Numbers indicate stars in the MACHO catalogue. Vertical lines indicate the theoretical ratio between fundamental $(\mathrm{F})$ and overtone $(1 \mathrm{O}$, first; $2 \mathrm{O}$, second) radial modes.

\subsection{The $F / 1 O=0.77$ ratio}

In the case of multimode HADS stars, it is quite obvious to search for the ratio 0.77 , i.e., the theoretical $\mathrm{F} / 1 \mathrm{O}$ ratio. At the beginning of the investigation on variability in the lower part of the instability strip, such a search was a misleading factor in the frequency analysis of the low-amplitude $\delta$ Sct stars, as their pulsational contents are actually more and more complicated. As a matter of fact, the 0.77 value was found between different terms solving the light curve of 44 Tau (Poretti et al. 1992), demonstrating how nonradial modes can also originate it. On the other hand, such a ratio has been found in several highamplitude radial pulsators (SX Phe, AE UMa, RV Ari, BP Peg and AI Vel).

In the V974 Oph data, the two frequencies $f_{1}$ and $f_{3}$ give $f_{1} / f_{3}=0.786$, which appears a little higher value, but similar to those found in MACHO 121.22427.551 (0.783) and MACHO 114.20368 .797 (0.790). V1162 Ori also supplies a still higher value (0.795). The period ratios are very accurate: in the case of the shortest time baseline, i.e. V974 Oph itself, the small errors on the frequencies propagate on an error bar of $\sim 1 \times 10^{-4}$ on the ratio. The $f_{1} / f_{3}$ ratio raises some theoretical problems. On one side, if $f_{1} / f_{3}$ is recognized as the fingerprint of the $\mathrm{F} / 1 \mathrm{O}$ ratio, V974 Oph is an interesting laboratory to study the effects of the rotation and/or the metallicity. Indeed, the high value requires a very low metallicity $(Z<0.0005$; see Fig. 3 in Petersen $\&$ Christensen-Dalsgaard 1996) and this seems to be unlikely. Fast rotation can account for this value (Pérez Hernández et al. 1995). However, some spectra taken with the Siding Spring 2.3-m telescope show very narrow metallic lines, setting an 
upper limit of a few $\mathrm{km} \mathrm{s}^{-1}$ (Kiss, private communication); hence, we can rule out fast rotation. On the other hand, if $f_{3}$ is a nonradial mode, the resonance effects $\left(f_{1} / f_{2}\right.$ and $\left.f_{1} / f_{3}\right)$ are an intriguing theoretical point.

\subsection{The 0.800 ratio}

The 0.800 ratio is found in double mode Cepheids pulsating in the 10 and 20 radial modes (Beltrame \& Poretti 2002). Such a value is also observed in some double-mode HADS stars (Musazzi et al. 1998) and it is attributed to the same modes. In the case of V974 Oph, we obtain $f_{1} / f_{4}=0.807$. However, if we accept the previous identification (i.e., $f_{1} / f_{3}=\mathrm{F} / 1 \mathrm{O}$ ), we must consider $f_{4}$ as a nonradial mode, as it has an intermediate value between the frequencies of the $\mathrm{F}$ and 10 modes. As a couple of values significantly different from 0.800 are found in MACHO 109.20634.24, the nonradial nature of some of these modes seems assured.

\section{Conclusions}

V974 Oph can be considered a unique high amplitude pulsating star in the current observational scenario:

1. The $f_{1} / f_{2}$ doublet makes V974 Oph similar to lowamplitude $\delta$ Sct and SX Phe stars from short-period side and to RR Lyr variables from the long-period side. No other star with a period around $0.20 \mathrm{~d}$ shows such a feature.

2. The $f_{1} / f_{3}$ ratio seems a bit higher than expected for $\mathrm{F}$ and 10 radial modes. A detailed spectroscopic analysis could clarify this point, via an evaluation of the influence of the metallicity or the detection of line profile variations suggesting nonradial modes.

3. Only a large number of terms could reduce the observed scatter. This finding looks very attractive for asteroseismic purposes, as it suggests the excitation of nonradial modes, whose presence was not so evident in other HADS stars.

In a certain sense, V974 Oph provides a link between low- and high-amplitude variables.

The identification of $f_{3}$ as a nonradial mode is also suggested by the presence of numerous cases of stars with frequency ratios greater (see Sect. 4.2) and smaller (MACHO 104.20389.1202 and MACHO 104.20638.40, Fig. 5) than the canonical $0.77-0.78$ value. As in the case of the LADS, we should not consider such a value uniquely as the signature of radial modes. If we also consider the cases with ratio around 0.800 , we can see how a large range of frequency ratios is actually observed, as expected from the excitation of a large variety of nonradial modes. Figure 5 also shows that there are some HADS stars displaying a frequency ratio close to 0.56 . The most robust case is provided by the long-term photometry of AI Vel: in their discussion Walraven et al. (1992) ruled out that such a ratio can be explained by two radial modes.

In the hypothesis of nonradial modes, it is quite interesting to verify if FG Vir, i.e., a case study in the $\delta$ Sct scenario, displays the same ratios. The fundamental radial mode $\left(12.154 \mathrm{~d}^{-1}\right)$ is well-identified and a high number of nonradial mode is observed (Breger et al. 1999; Mantegazza \& Poretti 2002). Indeed, a doublet is observed, even if with a slightly lower value $(12.154 / 12.716=0.956)$ than that observed in V974 Oph and other HADS stars. No frequency couple matches the $0.77-0.78$ ratio; there are three frequencies $\left(21.052,21.232,21.551 \mathrm{~d}^{-1}\right)$ supplying a ratio in the $0.577-$ 0.564 range, but none close to the 0.561 value. Different evolutionary stages (FG Vir is close to the Terminal Age Main Sequence, HADS stars should be more evolved) can account for these differences. Anyway, it is evident that asteroseismology is not confined in low amplitude pulsators and HADS stars can provide useful, complementary observational facts to model the pulsation. On the basis of the new results, the characterisation of the light variability of HADS stars needs to be done in a deeper way in the future.

Acknowledgements. The research has made use of the SIMBAD database, operated at CDS, Strasbourg, France. J. Vialle improved the English form of the manuscript. The author wishes to thank J. O. Petersen for fruitful discussions at an early stage of this work, for further useful comments on a first draft of the manuscript and on some checks about frequency analysis and modelling. The author also wishes to thank Rafa Garrido for useful comments and Laszlo Kiss for the spectroscopic determination of the $v \sin i$ value.

\section{References}

Alcock, C., Allsman, R., Alves, D. R., et al. 2000a, ApJ, 536, 798 Alcock, C., Allsman, R., Alves, D. R., et al. 2000b, ApJ, 542, 257 Arentoft, T., Sterken, C., Handler, G., et al. 2001, A\&A, 374, 1056 Beltrame, M., \& Poretti, E. 2002, A\&A, 386, L9

Breger, M. 1979, PASP, 91, 5

Breger, M., \& Bischof, K. 2002, A\&A, 385, 537

Breger, M., Pamyatnykh, A. A., Pikall, H., \& Garrido, R. 1999, A\&A, 341,151

Carpino, M., Milani, A., \& Nobili, A. M. 1987, A\&A, 181, 182

Garrido, R., \& Rodríguez, E. 1996, MNRAS, 281, 696

Kiss, L. L., Derekas, A., Mészáros, Sz., \& Székely, P. 2002, A\&A, 394, 943

Kuschnig, R., Weiss, W. W., Gruber, R., Bely, P. Y., \& Jenkner, H. 1997, A\&A, 328, 544

Mantegazza, L., \& Poretti, E. 2002, A\&A, 396, 911

Mazur, B., Krzemiński, W., \& Thompson, I. B. 2003, MNRAS, 340, 1205

McNamara, D. H. 2000, in Delta Scuti and Related Stars, ed. M. Breger, \& M. H. Montgomery, ASP Conf. Ser., 210, 373

Montgomery, M. H., \& O’Donoghue, D. 1999, Delta Scuti Star Newsletter, 13, 28

Moskalik, P., \& Poretti, E. 2003, A\&A, 398, 213

Musazzi, F., Poretti, E., Covino, S., \& Arellano Ferro A. 1998, PASP, 110,1156

Pérez Hernández, F., Claret, A., \& Belmonte, J. A. 1995, A\&A, 295, 113

Petersen, J. O., \& Christensen-Dalsgaard, J. 1996, A\&A, 312, 463

Poretti, E. 1991, A\&A, 245, 136

Poretti, E., \& Antonello, E. 1988, A\&A, 199, 191

Poretti, E., Mantegazza, L., \& Riboni, E. 1992, A\&A, 256, 113

Vaniček, P. 1971, Ap\&SS, 12, 10

Walraven, Th., Walraven, J., \& Balona, L. A. 1992, MNRAS, 254, 59

Zhou, A. Y. 2002, A\&A, 385, 503 